\title{
Zitationspraxen in deutschsprachigen Fachaufsatzeinleitungen
}

\author{
Karin Wetschanow (Wien)
}

\begin{abstract}
Since Swales' groundbreaking article (1990) on the rhetorical structure of research article introductions, a growing number of studies have explored the move structure of introductions. There is an enormous body of work analyzing citation practices in academic writing. Nevertheless, relatively little attention has been paid to the analysis of citation practices within different moves. This exploratory study of five German research articles investigates the relation of amount and types of citations within the different moves of research article introductions. The description and discussion of the five sample papers results in the following hypotheses: German research article introductions (1) can be divided in a territory-, a niche-, and a project-driven type, (2) prefer non-integral citations, (3) use indirect citations to establish a territory and (4) link the announced research to disciplinary fields using the instrument of direct terminology quotations.
\end{abstract}

\section{$1 \quad$ Einleitung}

Entsprechend ihrer zentralen Bedeutung für die Tradierung und den Austausch von Wissen innerhalb von wissenschaftlichen Diskursgemeinschaften, hat die Erforschung wissenschaftlicher Artikel innerhalb genretheoretischer Analysen in den letzten Jahrzehnten vielfach Beachtung gefunden. Insbesondere der Erforschung von in Fachzeitschriften publizierten Aufsätzen - sogenannten research articles (=RA) oder „Fachaufsätzen“ - kommt in der Genreanalyse ein großer Stellenwert zu. Mit John Swales (1981) bahnbrechender Analyse von einleitenden Textstellen wissenschaftlicher Zeitschriftenaufsätze ist dieser Abschnitt in den Mittelpunkt zahlreicher Arbeiten gerückt. Sein 1990 dargestelltes CARSModell (= Creating a Research Space) hat zu einer Reihe weiterer Analysen der rhetorischen Makrostruktur von Einleitungen motiviert. Untersucht wurden interkulturelle Unterschiede (vgl. Hirano 2009; Martín/Léon Pérez 2014) ebenso wie inter- (vgl. z. B.: Bruce 2014; Lin/Evans 2012; Samraj 2005; Tessuto 2015) und subdisziplinäre Variationen (vgl. z. B. Atai/Habibie 2012; Hyland 2000; Ling 2014; Ozturk 2007; Szurawitzki/Eiglsperger 2014) von einleitenden Textabschnitten.

Während einleitende Textteile in englischsprachigen Fachzeitschriftenaufsätzen recht häufig untersucht wurden und werden, ist die Zahl der Untersuchungen deutschsprachiger Daten erstaunlich gering. Die rezentesten Arbeiten hierzu stammen von Michael Szurawitzki: Er widmet sich in seinen Untersuchungen sowohl dem interkulturellen Vergleich mit finnischen Arbeiten (vgl. Szurawitzki 2011), der DAF-Perspektive (vgl. Szurawitzki 2014) aber auch den intradisziplinären Besonderheiten von medizinischen Fachzeitschriften (vgl. Szurawitzki/ 
Eiglsperger 2014). Sowohl Michael Szurawitzki als auch der Großteil der wenigen vorhandenen Arbeiten orientieren sich bei der Analyse der Makrostruktur an John Swales Analysemodell funktionaler Züge ('moves') (vgl. z. B.: Busch-Lauer 2001; Gnutzmann/Lange 1990; Thielmann 2009). Einige Arbeiten, wie Mikaela Petkova-Kessanlis (2009) und Maike Prestin (2011), versuchen funktional-pragmatische Kategoriensysteme zu entwickeln und zentrale „Teiltexthandlungen“ bzw. realisierte „Sprechhandlungen“ einleitender Textteile zu systematisieren, im Grunde dominiert aber John Swales moveAnalyse auch die Arbeiten zu deutschsprachigen Artikeln.

Eine von John Swales (1981, 1993, 2004) behauptete zentrale Funktion von Fachaufsatzeinleitungen ist die Anbindung der eigenen Forschung an bereits vorhandene Forschung. Damit rückt die Intertextualität als wichtiger Bestandteil von Einleitungen ins Zentrum der Aufmerksamkeit. Neben der rein numerischen Zählung von Zitationen zum Zweck der Evaluierung (bibliometrische Methoden) rücken immer mehr auch die inhaltsanalytische Analyse der kontextuellen Einbettung von Zitaten sowie die diskursanalytische Untersuchung der Form und Funktion von Zitaten in den Blick (vgl. zur Diskussion der Entwicklung der einzelnen Beschäftigungsfelder Okamura 2008). Für die vorliegende Arbeit ist insbesondere die diskursanalytische Herangehensweise von Interesse, da sie Aufschluss über die Funktion intertextueller Bezüge gibt. Gegenstand diskursanalytischer Untersuchungen sind Praktiken des Zitierens in wissenschaftlichen Publikationen und Arbeiten im akademischen Ausbildungskontext. Die Palette reicht in diesem Bereich von allgemeinen Systematisierungen des Zitierens in der englischen Wissenschaftspraxis (vgl. Swales 1986) über das Suchen nach disziplinären (vgl. z. B.: Charles 2006; Harwood 2009; Hu/Wang 2014; Hyland 1999) und interkulturellen Unterschieden (vgl. z. B.: Davis 2013; Flowerdew/Li 2007; Jalilifar/Dabbi 2012; Okamura 2008) bis hin zur Analyse der Zitierpraxis in verschiedenen Stufen des Erwerbs wissenschaftlicher Schreibkompetenz (vgl. z. B.: Mansourizadeh/Ahmad 2011; Petrić 2007; Samraj 2008, 2013; Shi 2010; Thompson 2005). Für den deutschsprachigen Raum sind insbesondere die Arbeiten von Jakobs (vgl. 1999, 2003) und die Arbeit von Thomas Griffig (2006) zu englisch- und deutschsprachigen linguistischen Fachaufsätzen von Interesse.

Obwohl die Zitationsanalyse auch in der Diskursanalyse einen breiten Raum einnimmt (vgl. z. B.: Hyland 1999; Jakobs 1999, 2003; Samraj 2013; Swales 1986; White 2004), wurden Funktionen und Formen intertextueller Verweise selten in Relation zu den funktionalen Abschnitten, in denen sie auftreten, untersucht. Lediglich die Arbeit von Alireza Jalilifar und Razieh Dabbi (2012) und die Arbeit von Betty Samraj (2008) untersuchen ganz dezidiert die Zitationspraxen in der Einleitung, sind aber mit ihrer Fokussetzung auf MAThesen wenig aussagekräftig im Hinblick auf die deutschsprachige professionelle Schreibpraxis.

Der vorliegende Beitrag widmet sich eben dieser Lücke und setzt es sich zum Ziel Zitationsund Genreanalyse von deutschsprachigen Fachaufsatzeinleitungen miteinander zu verbinden. Es wird der Frage nachgegangen, welche funktionalen Züge in deutschsprachigen Einleitungen realisiert werden und welche Formen intertextueller Bezüge in diesen Zügen auftreten. Analysiert werden fünf linguistische Fachzeitschriftenartikel, die nach formalpragmatischen Kriterien ausgesucht wurden. Die vorliegende qualitative Analyse verfolgt das 
Ziel, einen Einblick in die Zitierpraxis deutschsprachiger RA-Einleitungen zu geben und Hypothesen für weitere Untersuchungen zu entwickeln.

\section{$2 \quad$ Studie}

Für die qualitative Untersuchung wurden fünf Artikel aus deutschsprachigen linguistischen Fachzeitschriften herangezogen (vgl. Tab. 1). Für die Auswahl wurden pragmatische und strukturelle Auswahlkriterien angelegt: Aus der Liste von Fachzeitschriften, die zum Erhebungszeitpunkt unter dem Schlagwort Sprach- und Literaturwissenschaft im OnlineKatalog der Universitätsbibliothek der Universität Wien aufgelistet waren, wurden diejenigen ausgewählt, deren Publikationssprache vorrangig Deutsch ist. Aus dieser Liste wurden Fachzeitschriften gewählt, die originär linguistisch ausgerichtet sind, auf deren Volltexte mir zum Zeitpunkt der Erhebung aufgrund meiner institutionellen Anbindung an die Universität Wien der kostenlose Zugriff erlaubt war, und denen ein gewisser Bekanntheitsgrad innerhalb der Linguistik unterstellt werden kann. Als Maßeinheiten für den Bekanntheitsgrad zählten neben der subjektiven Einschätzung folgende Kriterien: Die Zeitschriften mussten (a) in relevanten Services (MLA International Bibliography etc.) indiziert (ZfAL, Sociolinguistica, ZGL) und/oder (b) mit linguistische Vereinigungen assoziiert (ZfAL, Gesprächsforschung) sein. Linguistic Online und Gesprächsforschung wurden als Repräsentantinnen der Open Access Journals ausgewählt.

Die Artikelauswahl wurde durch ein zeitliches und ein strukturelles Kriterium gesteuert: Aus der letzten verfügbaren Ausgabe jeder Fachzeitschrift wurde jeweils der erste Artikel herangezogen, der eine explizit als solche gekennzeichnete „Einleitung“ enthält. In einzelnen Fällen (ZfAL) musste um einen Jahrgang zurückgegangen werden, um eine solche Arbeit zu finden.

\begin{tabular}{|c|c|}
\hline Artikel & Abkürzung \\
\hline $\begin{array}{l}\text { Anders, Christina Ada/Palliwoda, Nicole/Schröder, Saskia (2014): „’'in dem } \\
\text { moment wo ich es dann erkenne dann ist es auch gleich wieder weg' - } \\
\text { Salienzeffekte in der Sprachperzeption“. Linguistik Online 66/4: 51-69. }\end{array}$ & $\begin{array}{l}\text { LO-Anders et } \\
\quad \text { al. }\end{array}$ \\
\hline $\begin{array}{l}\text { Bücker, Jörg (2013): „Sinngebiete lebensweltlicher Erfahrung in der SMS- } \\
\text { und Usenet-Kommunikation: Ein Vergleich“. Zeitschrift für angewandte } \\
\text { Linguistik 59/1: 21-19. }\end{array}$ & ZfAl-Bückner \\
\hline $\begin{array}{l}\text { Lindemann, Katrin/Ruoss, Emanuel/Weinzinger, Caroline (2014): } \\
\text { „Dialogizität und sequenzielle Verdichtung in der Forenkommunikation: } \\
\text { Editieren als kommunikatives Verfahren“. Zeitschrift für germanistische } \\
\text { Linguistik 42/2: 223-252. }\end{array}$ & $\begin{array}{l}\text { ZGL- } \\
\text { Lindemann et } \\
\quad \text { al. }\end{array}$ \\
\hline $\begin{array}{l}\text { Petkova, Mikaela (2012): „Der Kreislauf der Kontaktphänomene“. } \\
\text { Sociolinguistica 26/1: 1-17. }\end{array}$ & SL-Petkova \\
\hline $\begin{array}{l}\text { Zima, E. (2014): „Gibt es multimodale Konstruktionen? Eine Studie zu } \\
\text { [V(motion) in circles] und [all the way from X PREP Y]“. } \\
\text { Gesprächsforschung - Online: Zeitschrift zur verbalen Interaktion 15: 1-48. }\end{array}$ & GF-Zima \\
\hline
\end{tabular}

Tabelle 1: Übersicht über Zeitschriften und Artikel nach Autor_innennamen geordnet 
Für die Analyse der rhetorischen Makrostruktur der Einleitungen wird das Swale'sche CARSModell, wie es 1990 dargestellt wurde, gewählt (vgl. Tabelle 2). Dieses Modell wurde bereits für die Analysen deutschsprachiger wissenschaftlicher Einleitungen eingesetzt (vgl. BuschLauer 2001; Gnutzmann/Lange 1990; Petkova-Kessanlis 2009; Szurawitzki/Eiglsperger 2014), womit die Möglichkeit der Vergleichbarkeit gegeben ist. Zudem gewährleistet dieses genretheoretische Analysemodell eine Anbindung an die Erforschung englischsprachiger research articles, welche für den Bereich des English for specific purposes von großer Relevanz ist. Ein weiteres Argument für das Swale'sche Modell liegt in dessen zunehmend orientierungsgebender Funktion, die in der Zirkularität von Analyse- und Anwendungsmodell begründet liegt: Was als Modell anhand empirischer Daten im Analyseprozess entwickelt wurde, wird in der Schreibdidaktik mehr und mehr als Anleitungsmodell ein- und umgesetzt (vgl. etwa Huemer/Rheindorf/Gruber 2012).

\begin{tabular}{|l|l|l|}
\hline Move 1 & Establishing a territory & Territorium etablieren \\
\hline Step 1 & Claiming centrality and/or & Zentralität behaupten und/oder \\
\hline Step 2 & $\begin{array}{l}\text { Making topic generalisation(s) } \\
\text { and/or }\end{array}$ & $\begin{array}{l}\text { Thema: Verallgemeinerungen } \\
\text { und/oder }\end{array}$ \\
\hline Step 3 & $\begin{array}{l}\text { Reviewing items of previous } \\
\text { research }\end{array}$ & Bisherige Forschung besprechen \\
\hline Move 2 & Establishing a niche & Nische etablieren \\
\hline Step 1A & Counter-claiming or & Widersprechen oder \\
\hline Step 1B & Indicating a gap or & Lücke aufzeigen oder \\
\hline Step 1C & Question-raising or & Frage aufbringen oder \\
\hline Step 1D & Continuing a tradition & Tradition weiterführen \\
\hline Move 3 & Occupying the niche & Nische besetzen \\
\hline Step 1A & Outlining purposes or & Zwecke aufzeigen oder \\
\hline Step 1B & Announcing present research & Aktuelle Forschung ankündigen \\
\hline Step 2 & Announcing principal findings & Wichtigste Resultate ankündigen \\
\hline Step 3 & Indicating RA structure & RA-Struktur vorstellen \\
\hline
\end{tabular}

Tabelle 2: moves der Einleitung nach dem CARS-Modell (Swales 1990: 80)1

Für die Analyse der Zitiertätigkeit in den untersuchten Einleitungen wird Eva-Maria Jakobs (1999: 54) Auffassung von Zitaten als der ,sprachlich markierten Bezugnahme auf andere Texte“ übernommen und als „Form der markierten Intertextualität“ bezeichnet. Jakobs fasst mit ihrer Bezeichnung sehr beschreibend und wenig interpretierend alle als Handlungsaufforderungen oder Angebote gekennzeichneten Verweise auf textexterne

\footnotetext{
${ }^{1}$ In Swales (2004) wird die strikt lineare Reihenfolge des Modells mehr oder weniger aufgegeben und die dortigen Angaben fürs Englische ähneln damit ein wenig den in den wenigen empirischen Untersuchungen des Deutschen vorgeschlagenen Adaptionen.
} 
Textstellen zusammen. Realisiert sind solche Verweise als sprachliche oder semiotische Zeigehandlung. Laut Eva-Maria Jakobs (1999: 100) fungieren intertextuelle Verweise ,primär als (Such-) Anweisungen, wo bestimmte Informationen in anderen Texten zu finden sind, sowie als Handlungsaufforderung, vorhandene Wissensbestände zu reaktivieren, dieses Wissen zum sprachlichen Kontext des Verweises in Beziehung zu setzen und in dessen Interpretation einzubeziehen“. Verweise sind demnach das textuelle „Ergebnis“ einer Zeigehandlung und Angebote zur Sinnkonstruktion an die Leser_in. Zwei Momente finden sich dabei in allen Formen von intertextuellen Verweisen notwendigerweise wieder: etwas (textexternes), auf das verwiesen wird (Zitate) und sprachliche Mittel, mit denen verwiesen wird (Quellverweise). Um solche markierten Formen der Intertextualität systematisch untersuchen zu können, bedarf es eines Analyseinstrumentariums für die Formen von Zitaten und eines für die Beziehung zwischen Zitat(typ) und Quellverweis(typ).

Mit John Swales (1986) können Zitate in „direkte“, d. h. wörtliche Widergaben fremder Texte und ,indirekte“, d. h. paraphrasierte Formen der Zusammenfassung fremden Gedankenguts, unterteilt werden. Direkte Zitate können eigenständige Wiederholungen ganzer Textpassagen sein, sie können aber auch syntaktisch in den eigenen Text integriert sein. In jedem Fall sind sie als Texte, die einem anderen Text entstammen, markiert. Die Markierung kann entweder durch gängige Satzzeichen wie das Anführungszeichen realisiert werden, oder durch typografische Mittel wie die Kursivsetzung bzw. eine andere Schriftart, oder aber auch durch rein formale Mittel wie durch die Absatzschaltung und Einrückung. Eine Untergruppe direkter Zitate, die Eva-Maria Jakobs vorschlägt, soll hier übernommen werden: Sie konzeptioniert das zitierende Anführen von Fachbegriffen, Theorien-, Modell,- oder Konzeptnamen als „Anspielung““.

Eine weitere wichtige Unterscheidung, die John Swales (1986) trifft, ist die nach dem Kriterium des „Berichtens“. Sowohl direkte als auch indirekte Zitate können mit einer berichtenden Phrase eingeleitet werden oder nicht. In berichtenden Zitationen werden berichtende Verben eingesetzt, um intertextuelle Erkenntnisse, Behauptungen usw. zu erwähnen:

Lediglich Langacker (2001, 2005, 2008) argumentiert im Rahmen seiner Kognitiven Grammatik zumindest theoretisch explizit für die Möglichkeit der Entstehung bzw. Existenz multimodaler Symbole (Langacker 2005:104).

(GF-Zima)

Nicht-berichtende wörtliche, d. h. direkte Zitate, wären alle Blockzitate, die ohne jegliche berichtende Phrase (X behauptet, sagt, meint, etc...) in den Text eingebaut werden.

In der Analyse der berichtenden Zitationen sind zwei Fragen von weiterem Interesse: Wer handelt? Welche Handlung wird den zitierten Autor_innen zugeschrieben? Um diese Frage zu beantworten wird zwischen individualisierten und kollektivierten Akteur_innen unterschieden. Mit dem Begriff „Kollektivierung“ wird die meist metonymische Bezeichnung der Akteur_innen von Sprechhandlungen oder kognitiven Prozessen gefasst. In diese Kategorie fallen pauschale Erwähnungen von Texten (die Beiträge), Personen (Monetaristen wie...), Theorien oder Ideologien (die ökonomische Theorie), Disziplinen (in einer geschichtswissenschaftlichen Arbeit wird man...), soziale Felder (die Wissenschaft) oder auch Kulturen bzw. Kulturkreise (in der westlichen Welt). 
Mit der Zitation einher geht in der wissenschaftlichen Zitationspraxis das Erfordernis eines Quellverweises um die Nachvollziehbarkeit zu gewährleisten. In Anlehnung an Paul Thompson und Chris Tribble (2001) wurde in Auseinandersetzung mit dem Datenmaterial das folgende Analyseraster entwickelt: Primär wird zwischen ,integrierten“ und „nichtintegrierten Quellverweisen“ unterschieden.

Integrierte Quellverweise sind semantisch-syntaktisch in die intertextuelle Verweisstelle (das Zitat) eingebaut und können in die Kategorien „Quellverweis als Teil eines Werknamens“ und „nichtverweisende Quellangaben“ unterschieden werden. Ist der Quellverweis Teil eines Werknamens, wird der Quellverweis in eine semantisch-syntaktische Konstruktion eingebaut, hat Agensqualität und bildet gemeinsam mit dem Namen eine Einheit, die auf ein Werk referiert:

Wie Dürnscheids $(1999,2002 b)$ Untersuchungen [...].

(ZfAL-Bücker)

Fällt die Nennung der Jahreszahl in Klammer weg, so handelt es sich prinzipiell um eine „nichtverweisende Quellangabe“.

[...] Perspektive im Sinne Schütz', Luckmanns und Bergers.

(ZfAL-Bücker)

Nicht-integrierte Quellverweise sind semantisch-syntaktisch nicht in den Text integriert und können unterschiedliche Formen haben. Prinzipiell besteht ihr vorrangiges kommunikatives Ziel darin, eine vorab im Text getätigte Behauptung zu belegen. Beim „Beleg“ wird eine Behauptung durch einen Verweis auf eine externe Quelle belegt:

Die gruppeninternen Foren erscheinen so als kommunikative Mikrokosmen, [...] (vgl. Münker 2009).

(GF-Zima)

Die „,beispielhafte Nennung“ ist naturgemäß eng mit dem Beleg verbunden, unterscheidet sich aber insofern vom Beleg, als hier im Text Aussagen von anderen Autor_innen zusammenfassend paraphrasiert und einer Gruppe von Personen, Texten, Untersuchungen etc. zugeschrieben werden. Die beispielhafte Nennung kann entweder als Beleg für eine kollektivierende Phrase auftreten oder als expliziter Verweis auf beispielhaft genannte Quellen, die eine vorangegangene Paraphrase belegen. In beiden Fällen besteht die Funktion des Quellverweises darin, zusammenfassende Paraphrasen durch beispielhafte Nennung einiger konkreter Werke „fassbarer“ und damit nachvollziehbarer zu machen.

[...] wie dies neuere Arbeiten der Textlinguistik nahelegen (vgl. z.B. Roth/Pitzmüller 2007; Fix 2008; Hausendorf/Kesselheim 2008), [...].

(GF-Zima)

Eine besondere Form stellen das syntaktisch integrierte und auch das freistehende ,direkte Zitat“ und die „Anspielung“ - also das wörtliche Zitat einer Begrifflichkeit oder eines konkreten Ausdrucks - dar. Sie geben die Aussage einer anderen Person(engruppe) wider und setzen deren Stimme anstelle der eigenen. Streng genommen kommt daher dieser Art des Quellverweises auch die Funktion der „Stimmabgabe“ zu, denn man lässt eine andere Person sprechen. 
Seitdem ist das Thema, wie Gardner-Chloros (2009: 10) in ihrem Überblick feststellt, „,endlos diskutiert" worden, [...].

(LO-Anders et al.)

Belegt kann aber auch die Erwähnung oder Schaffung eines Begriffs, eines beobachteten Phänomens, eines wissenschaftlichen Konzepts, einer Theorie oder Methodologie werden. Bei dieser Art des Belegs handelt es sich im Grunde um die Quellangabe für eine besondere Form des direkten Zitats, nämlich um die Quellangabe für eine Anspielung:

Diese Konstruktion beschreibt eine bestimmte Art der Bewegung (,manner of motion', Talmy 2000) bzw. eine spezifische , path shape' (Vulchanova/Martinez 2013), nämlich zirkuläre Bewegung.

(GF-Zima)

Für die Untersuchung der Zitationspraxis in deutschsprachigen Fachzeitschrifteneinleitungen wird entsprechend der oben erläuterten Kategorien folgendes Analyseraster festgelegt:

\begin{tabular}{|c|c|}
\hline \multicolumn{2}{|r|}{ Funktionale Abschnitte } \\
\hline Territorium etablieren & $\begin{array}{l}\text { Zentralität behaupten } \\
\text { Thema nennen: Verallgemeinerungen } \\
\text { bisherige Forschung besprechen }\end{array}$ \\
\hline Nische etablieren & $\begin{array}{l}\text { Widersprechen } \\
\text { Lücke aufzeigen } \\
\text { Frage aufbringen } \\
\text { Tradition weiterführen }\end{array}$ \\
\hline Nische besetzen & $\begin{array}{l}\text { Zwecke aufzeigen } \\
\text { aktuelle Forschung ankündigen } \\
\text { wichtigste Resultate ankündigen } \\
\text { RA-Struktur zeigen }\end{array}$ \\
\hline \multicolumn{2}{|r|}{ intertextuelle Verweistypen } \\
\hline $\begin{array}{l}\text { Direkte Zitate/ Anspielungen } \\
\text { Indirekte Zitate }\end{array}$ & \\
\hline \multicolumn{2}{|r|}{ Berichtstyp } \\
\hline $\begin{array}{l}\text { berichtend } \\
\text { nicht-berichtend }\end{array}$ & \\
\hline \multicolumn{2}{|r|}{ Akteur_innentypen } \\
\hline $\begin{array}{l}\text { Individuelle Akteur_innen } \\
\text { Kollektivierte Akteur_innen }\end{array}$ & \\
\hline \multicolumn{2}{|c|}{ Funktion des Quellverweises } \\
\hline Integrierte Quellverweise & $\begin{array}{l}\text { Quellverweis als Teil eines Werknamens: } \\
\text { Nichtverweisende „Quellangabe“ }\end{array}$ \\
\hline $\begin{array}{l}\text { Nicht-integrierte } \\
\text { Quellverweise }\end{array}$ & $\begin{array}{l}\text { Beleg } \\
\text { Beispielhafte Nennung } \\
\text { Quellangabe }\end{array}$ \\
\hline
\end{tabular}

Tabelle 3: Analyseraster 


\section{$3 \quad$ Ergebnisse}

\subsection{Funktionale Abschnitte in den Einleitungen}

In einem ersten Schritt werden die einleitenden Textstellen den drei funktionalen Abschnitten ('moves') und ihren Einzelschritten ('steps' oder 'sub-moves') zugeordnet bzw. wenn nötig neue funktionale Abschnitte kategorisiert. In einem nächsten Schritt wird die Wortanzahl pro funktionalem Abschnitt ermittelt (vgl. Tab. 5), um den Vergleich der einzelnen Sequenzen auch auf der Ebene der Textmengen zu ermöglichen. Tabelle 4 gibt einen Überblick über realisierte Züge, Sub-Züge und Wortmengen pro Zug in den jeweiligen Einleitungen. Da neuere Untersuchungsergebnisse (vgl. Ling 2014) darauf hinweisen, dass das Vorhandensein eines nachfolgenden eigenen Literaturteils die rhetorische Makrostruktur der Einleitung beeinflusst, wird in diesem ersten Überblick auch darüber Auskunft gegeben, ob ein solcher Abschnitt in den untersuchten Artikeln vorhanden ist oder nicht.

\begin{tabular}{|c|c|c|c|c|c|}
\hline & LO-Anders et al. & ZfAL-Bücker & $\begin{array}{l}\text { ZGL- } \\
\text { Lindemann }\end{array}$ & SL-Petkova & GF-Zima \\
\hline \multirow{3}{*}{$\begin{array}{l}\text { Territoriu } \\
\text { m } \\
\text { etablieren }\end{array}$} & $\begin{array}{l}\text { Zentralität } \\
\text { behaupten }\end{array}$ & $\begin{array}{l}\text { Zentralität } \\
\text { behaupten }\end{array}$ & $\begin{array}{l}\text { Zentralität } \\
\text { behaupten }\end{array}$ & $\begin{array}{l}\text { Zentralität } \\
\text { behaupten }\end{array}$ & $\begin{array}{l}\text { Zentralität } \\
\text { behaupten }\end{array}$ \\
\hline & $\begin{array}{l}\text { Aktualität in der } \\
\text { Forschung }\end{array}$ & $\begin{array}{l}\text { Aktualität in der } \\
\text { Forschung }\end{array}$ & $\begin{array}{l}\text { Aktualität in } \\
\text { der } \\
\text { Gesellschaft }\end{array}$ & & $\begin{array}{l}\text { Aktualität in der } \\
\text { Forschung }\end{array}$ \\
\hline & Forschungsstand & & & Forschungsstand & \\
\hline $\begin{array}{l}\text { Nische } \\
\text { etablieren }\end{array}$ & $\begin{array}{l}\text { Lücke: } \\
\text { Datenmangel }\end{array}$ & $\begin{array}{l}\text { Lücke: } \\
\text { Perspektive }\end{array}$ & & & $\begin{array}{l}\text { Lücke: } \\
\text { Perspektive }\end{array}$ \\
\hline \multirow[t]{7}{*}{$\begin{array}{l}\text { Nische } \\
\text { besetzen }\end{array}$} & $\begin{array}{l}\text { aktuelle } \\
\text { Forschung } \\
\text { ankündigen }\end{array}$ & $\begin{array}{l}\text { aktuelle } \\
\text { Forschung } \\
\text { ankündigen }\end{array}$ & $\begin{array}{l}\text { aktuelle } \\
\text { Forschung } \\
\text { ankündigen }\end{array}$ & & $\begin{array}{l}\text { aktuelle } \\
\text { Forschung } \\
\text { ankündigen }\end{array}$ \\
\hline & & & Methodologie & & \\
\hline & & & Korpus & & \\
\hline & & & Vorgehen & & \\
\hline & $\begin{array}{l}\text { Zweck } \\
\text { aufzeigen }\end{array}$ & $\begin{array}{l}\text { Zweck } \\
\text { aufzeigen }\end{array}$ & $\begin{array}{l}\text { Zweck } \\
\text { aufzeigen }\end{array}$ & $\begin{array}{l}\text { Zweck } \\
\text { aufzeigen }\end{array}$ & \\
\hline & & $\begin{array}{l}\text { aktuelle } \\
\text { Forschung } \\
\text { ankündigen }\end{array}$ & & $\begin{array}{l}\text { aktuelle } \\
\text { Forschung } \\
\text { ankündigen }\end{array}$ & $\begin{array}{l}\text { Phänomen } \\
\text { auswahl }\end{array}$ \\
\hline & RA-Struktur & RA-Struktur & RA-Struktur & & $\begin{array}{l}\text { RA-Ende- } \\
\text { Struktur } \\
=\text { indirektes Ziel }\end{array}$ \\
\hline $\begin{array}{l}\text { Literaturtei } \\
\text { I } \\
\text { nachfolgen } \\
\text { d? }\end{array}$ & ja & nein & ja & nein & ja \\
\hline
\end{tabular}

Tabelle 4: moves und sub-moves der Einleitungen

Die vergleichende Analyse aller untersuchten Einleitungen macht deutlich, dass zwar alle Einleitungen in der einen oder anderen Form die funktionalen Abschnitte „Territorium etablieren" und "Nische besetzen" realisieren, dass jedoch nur in drei der untersuchten Artikel (LO-Anders et al., ZfAL-Bücker, GF-Zima) eine Nische etabliert wird. Die beiden 
anderen Aufsätze (ZGL-Lindemann et al., SL-Petkova) binden ohne Nennung eines Forschungsdesiderats das eigene Forschungsvorhaben an einen eingangs thematisierten Forschungsbereich an.

Die drei Arbeiten, die alle funktionalen Züge realisieren und dabei die von Swales in seinem CARS-Modell vorgeschlagene Reihenfolge einhalten, räumen der Ausformulierung der Lücke sehr unterschiedlich Platz ein: ZfAL-Bücker integriert die Forschungslücke in der Beschreibung relevanter Forschungszugänge durch einen mit der modalen Satzverbindung „ohne dass“ eingeleiteten Nebensatz:

In den letzten Jahren sind sowohl die Kommunikation via Short Message Service (SMS) als auch die Usenet-Kommunikation Gegenstand einer Reihe linguistischer Untersuchungen geworden [Territorium etablieren]], [ohne dass beide Kommunikationsformen vergleichend aufeinander bezogen worden wären [implizit etablierte Nische].

(ZfAL-Bücker)

LO-Anders et al. widmen dem Herausarbeiten der Lücke immerhin einen ganzen Satz und GF-Zima gar eine 432 Worte umfassende Textpassage, die drei längere Blockzitate beinhaltet. GF-Zima leitet ihre Forschungslücke in einem dreistufigen Verfahren ab: (1) In einem ersten Schritt identifiziert sie den von ihr genannten Forschungsbereich als einen, der in der relevanten Teildisziplin nicht behandelt wird (Absatz 1). (2) Anschließend referiert sie mit Paraphrasen und drei direkten Zitaten einen Kollegen, der ,zumindest theoretisch explizit" auf das untersuchte Problem hinweist (Absatz 2, 3). All diese Ausführungen führen GF-Zima schlussendlich erst zur Identifizierung der eigentlichen Lücke, nämlich zu der Feststellung, dass datenbasierte Ausarbeitungen fehlen (Absatz 4).

Dieser sehr elaborierten Herleitung eines Forschungsdesiderats diametral entgegengesetzt steht der Fachaufsatz ZGL-Lindemann et al. Diese Fachaufsatzeinleitung kommt gänzlich ohne Erwähnung einer Lücke oder die Identifizierung eines Forschungsdesiderats aus. In nur dreieinhalb Zeilen (= 27 Wörter) der insgesamt 41 Zeilen (= 645 Wörter) langen Einleitung wird das Forschungsfeld etabliert, der Rest der Einleitung dient dazu, das eigene Forschungsvorhaben detailliert vorzustellen und zu beschreiben. Trotz der Erwähnung einer Forschungslücke weist die Einleitung von ZfAL-Bücker eine ähnliche Struktur auf: Auch dieser Autor etabliert das Territorium in den zwei ersten Sätzen der Einleitung, um dann zur Beschreibung seines Vorhabens überzugehen, wobei diese immer wieder von Bezügen auf vorhandene Forschung durchzogen ist. Der letzte Absatz der Einleitung besteht aus einer expliziten, sehr konkreten theoretischen und methodischen Verortung, in der auf konkrete Werke Bezug genommen wird, und der mit einer Beschreibung des Ziels und des Aufbaus der Arbeit endet.

Eine wiederum gänzlich andere Fokussierung zeigt sich in der Einleitung von SL-Petkova. Die zentrale Funktion dieser Einleitung scheint die Forschungsanbindung zu sein. Selbst die Besetzung der nicht explizit genannten „Lücke“ ist bei dieser Autorin von Bezugnahmen auf bestehende Forschung gekennzeichnet: SL-Petkova identifiziert zwar implizit in der Zusammenfassung bisheriger Forschungsergebnisse eine Lücke, betont aber, dass sie diese nicht schließen sondern weiterführen wird: 
[...],,endlos diskutiert worden, ohne dass grössere [sic!] Einigkeit erreicht worden wäre“. Auch dieser Beitrag kann sich nicht zum Ziel setzen, zur Vereinheitlichung der verschiedenen Forschungsansätze oder der terminologischen Begrifflichkeiten beizutragen“".

(SL-Petkova)

Das Vorhandensein aller zentralen Elemente einer Einleitung, wie sie Swales beschreibt, hängt im untersuchten Material nicht mit der Länge eines Textes zusammen (vgl. Tab. 5). Im Gegenteil repräsentiert gerade die kürzeste Einleitung (LO-Anders et al.) eine relativ symmetrische Realisierung aller funktionaler Textabschnitte: Die Textmenge, die aufgewandt wird, um das Forschungsfeld zu etablieren und jene, die gebraucht wird um die Nische zu besetzen, halten einander annähernd die Waage. Die Forschungsnische als Dreh- und Angelpunkt zwischen den beiden Teilen ist nicht übermäßig lang, wird aber mit einer Länge von $8 \mathrm{Wörtern}$ ausreichend wahrgenommen.

\begin{tabular}{|l|r|r|r|r|r|}
\hline & $\begin{array}{c}\text { LO-Anders } \\
\text { et al. }\end{array}$ & ZfAL-Bücker & $\begin{array}{c}\text { ZGL- } \\
\text { Lindemann }\end{array}$ & $\begin{array}{c}\text { SL- } \\
\text { Petkova }\end{array}$ & GF-Zima \\
\hline \multicolumn{7}{|c|}{ Territorium etablieren } \\
\hline Wortanzahl & 82 & 57 & 27 & 312 & 99 \\
\hline i. Verweise & 3 & 0 & 0 & 8 & $1^{2}$ \\
\hline Anzahl Qu. & 6 & 0 & 0 & 12 & 0 \\
\hline \multicolumn{7}{|c|}{ Nische etablieren } \\
\hline Wortanzahl & 18 & \multicolumn{7}{|c|}{0} & 0 & 50 & 429 \\
\hline i. Verweise & 0 & 0 & 0 & 2 & 9 \\
\hline Anzahl Qu. & 0 & 0 & 0 & 2 & 7 \\
\hline \multicolumn{7}{|c|}{ Nische besetzen } \\
\hline Wortanzahl & 114 & 348 & 645 & 192 & 288 \\
\hline i. Verweise & 0 & 11 & 9 & 2 & 2 \\
\hline Anzahl Qu. & 0 & 16 & 9 & 2 & 2 \\
\hline
\end{tabular}

Tabelle 5: Anzahl intertextueller Verweise (=i. Verweise) und zitierter Quellen (=Anzahl Qu.) in einzelnen moves $^{3}$

Die untersuchten Artikel können entsprechend ihrer Fokussierung auf bestimmte Züge in „territoriumslastige“, „nischenlastige“ und „vorhabenslastige“ unterschieden werden. Im Datenmaterial weisen drei der untersuchten Fachaufsatzeinleitungen (LO-Anders et al., ZfAL-Bücker, ZGL_Lindemann et al.) eine „vorhabenslastige“ Struktur auf, d. h. sie räumen der Präsentation des aktuellen Forschungsvorhabens den meisten Raum ein. Konträr dazu kann die Einleitung Petkovas als „territoriumslastig“ bezeichnet werden: Mit ihren häufigen Bezügen auf Forschungszugänge und bisherige Arbeiten setzt die Autorin die Verortung der eigenen Arbeit im Forschungszusammenhang ins Zentrum der Aufmerksamkeit. Während der Etablierung einer Nische im untersuchten Datenmaterial tendenziell wenig Raum gegeben wird, steht bei GF-Zimas Einleitung das Herausarbeiten einer Forschungslücke im Zentrum. Ihre Einleitung könnte als ,nischenlastig“ bezeichnet werden.

\footnotetext{
${ }^{2}$ Hierbei handelt es sich um eine kollektivierende Phrase, der kein Beleg durch einen Quellverweis folgt.

${ }^{3}$ Als Einheit wurde jeweils die intertextuelle Verweistätigkeit gewählt: Ein Quellverweis etwa, der als Beleg einer kollektivierenden Phrase dient, wird als eine Einheit gewertet, obwohl in einem solchen Verweis mehrere Quellen zitiert sein können. Die kollektivierende Phrase (z. B.: „,neuere Untersuchungen“) und der Beleg wird ebenfalls als zusammengehörig erachtet und damit als EINE intertextuelle Verweistätigkeit gezählt. Ebenso wird ein wörtliches Zitat plus dazugehörigem Quellverweis als eine einzige intertextuelle Verweistätigkeit gewertet.
} 


\subsection{Intertextuelle Verweistätigkeit in den funktionalen Abschnitten}

Geht man davon aus, dass die zentrale Funktion wissenschaftlicher Intertextualität die Vernetzung mit bestehender Forschung und die Positionierung im Forschungsfeld ist, könnte man annehmen, dass in der ,territoriumslastigen“ Einleitung gegenüber der vorhabens- und lückenlastigen Einleitungen mehr intertextuelle Verweise auftreten. Die Analyse des Datenmaterials deutet aber vielmehr darauf hin, dass dies nicht der Fall ist: Im Datenmaterial wird keiner der möglichen funktionalen Abschnitte bevorzugt für intertextuelle Verweistätigkeit genutzt.

Die jeweilige Dominanz eines Zuges in einer Einleitung geht einher mit einer entsprechenden Häufigkeit an intertextuellen Verweisen in diesem Zug: Ist eine Einleitung „territoriumslastig“, dann finden sich im Zug „Territorium etablieren“ die meisten Realisierungen markierter Intertextualität, fokussiert sie die Lücke, so finden wir im Zug „Nische etablieren“ die meisten intertextuellen Verweise und ist sie „vorhabenslastig“, so ist der funktionale Abschnitt „Nische besetzen“ verstärkt von Intertextualität durchzogen. Das erscheint oberflächlich als banale Feststellung, jedoch ist von der quantitativen Textmenge pro Zug nicht direkt auf die Häufigkeit des Einsatzes markierter Intertextualität zu schließen, denn aus dem Hauptfokus auf die Beschreibung des eigenen Forschungsprojekts kann nicht direkt auf den vermehrten Einsatz textexterner Quellen geschlossen werden. Das untersuchte Material legt allerdings den Schluss nahe, dass eine Fokussierung auch den Einsatz von intertextuellen Verweisen triggert.

Ein eindrückliches Beispiel, das diesen Zusammenhang belegt, ist die Einleitung von ZGLLindemann et al. Obwohl laut Funktion des Zuges „Territorium etablieren“ davon ausgegangen werden könnte, dass bei seiner Realisierung intertextuelle Verweise der einen oder anderen Form vorkommen, bleibt der Zug in dieser Einleitung völlig frei von markierter Intertextualität. Das untersuchte Phänomen wird ausschließlich als interessantes gesellschaftliches Phänomen präsentiert. Entsprechend der „Vorhabenslastigkeit“ dieser Einleitung ist auch die Erwähnung relevanter bisheriger Forschungsarbeiten in der Darstellung des Forschungsvorhabens integriert: Mit der Kollektivierung ,neuere Arbeiten der Textlinguistik“ wird die Aktualität des eigenen Vorhabens betont, die in Klammern angeführten Verweise auf drei rezente Werke, soll die allgemeine Phrase belegen und damit die Behauptung nachvollziehbar und kontrollierbar machen:

[...] wie dies neuere Arbeiten der Textlinguistik nahelegen (vgl. z. B. Roth/Spitzmüller 2007; Fix 2008; Hausendorf/Kesselheim 2008), [...].

(ZGL-Lindemann et al.)

Ebenso lässt das Vorhandensein aller drei Züge nicht darauf schließen, dass in allen drei funktionalen Abschnitten auf textexterne Quellen verwiesen wird. Die Einleitung von LOAnders et al. etwa realisiert alle Züge in relativ ausgewogener Form, bedient sich aber nur im funktionalen Abschnitt „Territorium etablieren“ intertextueller Verweise, während GF-Zima in allen drei Zügen intertextuelle Verweise einsetzt.

Sehr gewagt, kann das indirekte Zitat als die präferierte Form der intertextuellen Verweistätigkeit im Zug „Territorium etablieren“ angenommen werden. Egal ob berichtend oder nicht-berichtend wird es gerne dazu eingesetzt, einen breiten Forschungsüberblick zu 
geben (LO-Anders et. al) oder konkrete untersuchungsrelevante Diskussionen von Begrifflichkeiten oder Konzepten (SL-Petkova) zusammenzufassen.

Als Prototyp dieser Form kann LO-Anders et al. angesehen werden. Diese Einleitung realisiert in relativ ausgewogener Form alle funktionalen Abschnitte einer Fachaufsatzeinleitung und entspricht damit am ehesten dem Swale'schen CARS-Model. Die Autorinnen dieser Einleitung setzen ihre intertextuellen Verweise dazu ein, um einen Überblick über rezente Forschung zu geben. Gleich der erste Satz der Einleitung thematisiert die wissenschaftliche Handlung ('research act') von nicht näher identifizierten Akteuren, den „neueren Untersuchungen“. Die solcherart unbestimmte, weil sich einer wissenschaftlich eingeforderten Nachvollziehbarkeit (vorerst) entziehende Behauptung, wird am Ende des Satzes durch in der Klammer gegebene beispielhafte Nennungen von vier Quellen aus ihrer Vagheit herausgeführt. Den intertextuellen Verweisen kommt die Funktion eines beispielhaften Belegs für eine kollektivierte Behauptung zu. Eine solche Form geht mit einer hohen Informationsdichte einher: Mehr als die Hälfte der insgesamt sechs angeführten Werke dienen dem Beleg dieser kollektivierenden Phrase innerhalb eines Quellverweises:

Neuere Untersuchungen haben gezeigt, dass [...] (cf. Kehrein 2012a, 2012b; Purschke 2012; Elmentaler/Gessinger/Wirrer 2010).

(LO-Anders et al.)

Die beiden weiteren Quellverweise in der Einleitung fungieren jeweils als beispielhafte Belegstellen für indirekte Zitate, die die Meinung einer wissenschaftlichen Community zusammenfassend wiedergegeben. Die Quellverweise dienen hier als Verweise auf beispielhaft ausgewählte Belege.

So ist etwa das palatale /s/ in süddeutschen regionalen Varietäten offenkundig ein TriggerMerkmal, das die regionale Zuordnung und Stereotypisierung auslöst (cf. Hundt 1992), während Merkmale wie die /r/-Vokalisierung in norddeutschen regionalen Varietäten als nicht-auffällige Variante der Standardsprache angesehen wird (cf. Elmentaler 2012).

(LO-Anders et al.)

Die stärkste Verbindung zwischen intertextueller Verweisform und funktionalem Abschnitt kann für die „Anspielung“ festgestellt werden. Anspielungen auf Begrifflichkeiten und Schulen sind in den untersuchten Einleitungen bis auf einen einzigen Beleg allesamt $(\mathrm{N}=14+$ 1) im Zug „Nische besetzen“ realisiert (lediglich SL-Petkova wählt diese Form des intertextuellen Verweises auch im funktionalen Abschnitt „Nische etablieren“). Der Grund hierfür dürfte in der Verwendung dieser Form und damit in ihrer Funktion begründet sein: Anspielungen dienen oft der Nennung konkreter Begrifflichkeiten mit Hilfe derer eine Vernetzung mit konkreten Theorien und Methoden hergestellt wird. Diese Konkretisierung entspricht demnach der Aufgabe des Zuges, das eigene Vorhaben möglichst konkret vorzustellen und in einem Theorie- und Methodennetz zu positionieren.

Die Einleitung von ZGL-Lindemann et al. etwa bedient sich im funktionalen Abschnitt „Nische besetzen“ der Anspielungen, um die eigene Arbeit theoretisch und methodisch in im Forschungsfeld $\mathrm{zu}$ verorten. Die Anspielungen ohne näheren Kontext alleine listenhaft angeführt bieten bereits einen guten Einblick in die methodische Verortung der Arbeit: 
„Sehfläche“ (Schmitz 2011)

„Möglichkeitsraum“ (Münker 2009: 57)

„medienspezifischen Potenzial““ (Bader/Fritz 2011: 59)

(ZGL-Lindemann et al.)

Ebenso nutzt ZfAL-Bücker, der ausschließlich im Zug „Nische etablieren“ auf textexterne Stellen verweist, die Form der Anspielung dazu, das eigene Vorhaben zu positionieren:

„,interpersonaler Kommunikation“ (Dürscheid 2007)

„geschlossenes Sinngebiet der alttäglichen Lebenswelt““ (Schütz/Luckmann 2003[1975])

„Online-Communities“" (vgl. u. a. Stegbauer 2001)

gesprächsanalytisch und interaktionallinguistisch vor (vgl. u. a. Selting/Couper-Kuhlen 2000,2001).

„getippten Dialoge“ bzw. „getippten Gespräche“ (Storrer 2001, 2002; Beißwenger 2002)

(ZfAL-Bücker)

Sowohl das semantisch-syntaktisch integrierte direkte Zitat als auch das Blockzitat werden in der Einleitung weitgehend vermieden. Lediglich die Einleitung von GF-Zima setzt Blockzitate ein, um eine Nische zu belegen und herzuleiten, alle anderen Einleitungen verzichten auf diese Form des intertextuellen Verweises. Das semantisch-syntaktisch integrierte direkte Zitat wird ebenfalls nur einmal eingesetzt: SL-Petkova setzt es ein, um sich auf formulativer Ebene zu entlasten, wenn sie abschließend für ihre Vorgangsweise argumentiert:

[...] wie Bell schreibt, „having two discrete languages [...]“ (Bell 1984: 176). Daher wird in diesem Beitrag von einem kontinuumsartigen Kreislauf der Phänomene gesprochen, die durch Kontakt zwischen verschiedenen Codes entstanden sind.

(SL-Petkova)

Bis auf die Einleitung von GF-Zima, die einen starken Fokus auf diesen Zug legt und mit einem Forschungsüberblick vermischt, scheint der funktionale Abschnitt „Nische etablieren“ eher frei von intertextuellen Verweisen. In der Regel werden zum Behaupten einer Nische keine intertextuellen Verweise eingesetzt. Wird zitiert, dann in Form von Anspielungen und Kurzzitaten, die auf der formulativen Ebene entlasten und gleichzeitig ein Argument für die behauptete Lücke liefern, wie bei SL-Petkova:

„,impossible task“" (Milroy/ Muysken 1995: 12).

Seitdem ist das Thema, wie Gardner-Chloros (2009: 10) in ihrem Überblick feststellt, „,endlos diskutiert" worden,

(SL-Petkova)

Die komplexe Herleitung einer Lücke, wie sie in GF-Zima vorliegt, weist ein hohes Maß an markierter Intertextualität auf. Entsprechend der Tatsache, dass die Etablierung der Nische in diesem Artikel einen zentralen Stellenwert einnimmt und in enger Auseinandersetzung mit der Literatur herausgearbeitet wird, finden sich nahezu alle Verweise der Einleitungen in diesem funktionalen Abschnitt. Die Wahl der Zitattypen ist insofern überraschend, als diese Einleitung die einzige im Datenmaterial ist, in der syntaktisch nicht integrierte Blockzitate eingebaut sind: im vorliegenden Fall sind es sogar drei. Innerhalb des Textes, in den die Blockzitate eingebettet sind, befinden sich wiederum Quellverweise, die auf weitere Werke des Autors der Blockzitate und auf einen Sammelband, in dem die Autorin veröffentlicht hat, verweisen. Alle drei Blockzitate werden durch Textstellen an den Text angebunden, die 
allesamt nach demselben Muster aufgebaut sind: Ein indirektes berichtendes Zitat, dessen Akteur der immergleiche referierte Autor (Langacker) ist, leitet das Blockzitat ein:

Lediglich Langacker (2001, 2005, 2008) argumentiert im Rahmen seiner Kognitiven Grammatik zumindest theoretisch explizit für die Möglichkeit der Entstehung bzw. Existenz multimodaler Symbole (Langacker 2005:104):

In Cognitive Grammar [...], the form in a form-meaning pairing is specifically phonological structure. I would of course generalize this to include other symbolizing media, notably gesture and writing.

Cognitive Grammar takes the straightforward position that any aspect of a usage event, or even a sequence of usage events in a discourse, is capable of emerging as a linguistic unit, should it be a recurrent commonality. (Langacker 2001:146, Hervorhebung im Original).

In einem kurzen Beitrag in einem Sammelband zu „Metaphor and Gesture“(Cien-ki/Müller 2008) geht er über dieses theoretische Bekenntnis sogar noch etwas hinaus (Langacker 2008:250):

When a baseball umpire yells Safe! and simultaneously gives the standard gestural signal to this effect (raising both arms together to shoulder level and then sweeping the hands outward, palms down), why should only the former be analyzed as part of the linguistic symbol? Why should a pointing gesture not be considered an optional component of a demonstrative's linguistic form?

Dabei gibt er allerdings innerhalb der Kognitiven Grammatik folgerichtig zu bedenken, dass nicht jede koverbal gebrauchte Geste automatisch als Teil der sprachlichen Einheit angenommen werden kann (Langacker 2008:250f.):

[...] It does not however follow that every gesture (that accompanies speech) has linguistic status. (...) A structure per se qualifies as an element of a language just to the extent that it is entrenched in the minds of the speakers and conventional in the speech community. [... However,] if a gesture is both familiar and conventional in a speech community, bearing a systematic relation to the expressions it occurs in, its exclusion from 'the language' would be arbitrary.

(GF-Zima)

Sieht man sich die Quellverweistypen näher an (vgl. Tab. 6), kann eindeutig eine Präferenz für die nicht-integrierten Quellverweise festgestellt werden.

\begin{tabular}{|l|c|c|c|c|c|}
\hline & $\begin{array}{c}\text { LO-Anders } \\
\text { et al. }\end{array}$ & $\begin{array}{r}\text { ZfAL- } \\
\text { Bücker }\end{array}$ & $\begin{array}{c}\text { ZGL- } \\
\text { Lindemann } \\
\text { et al. }\end{array}$ & SL-Petkova & GF-Zima \\
\hline Integrierte QU-V & & $\mathbf{6}$ & & $\mathbf{2}$ & $\mathbf{1}$ \\
\hline Werkname & 5 & & 2 & 1 \\
\hline nichtverweisend & $\mathbf{3}$ & $\mathbf{5}$ & $\mathbf{1 0}$ & $\mathbf{9}$ & $\mathbf{9}$ \\
\hline $\begin{array}{l}\text { Nicht-integrierte } \\
\text { QU-V }\end{array}$ & 3 & 2 & 2 & 1 & 1 \\
\hline Beleg & & 3 & 2 & 2 \\
\hline $\begin{array}{l}\text { Beispielhafte } \\
\text { Nennung }\end{array}$ & & & & & \\
\hline
\end{tabular}




\begin{tabular}{|l|l|l|l|l|l|}
\hline Quellangabe & & 3 & 4 & 6 & 6 \\
\hline
\end{tabular}

Tabelle 6: Quellverweistypen $(=Q U-V)$ in den untersuchten Einleitungen

Quellverweise dienen als Quellangaben zu direkten Zitaten, insbesondere zu den häufig vertretenen Anspielungen, zu nicht-berichtenden Paraphrasen und als beispielhafte Belege für verallgemeinernde Kollektivierungen. Selten werden Quellverweise in den Text integriert, im Datenmaterial setzt nur ZfAL-Bücker diese Strategie gehäuft ein:

Wie Dürnscheids $(1999,2002 b)$ vergleichende Untersuchungen zeigen [...].

Die vorliegende Untersuchung schließt $u$. a. an Günthners $(2011,2012)$ Untersuchungen zur SMS-Kommunikation als Dialog sowie an Beißwengers (i. Dr.) und Diekmannshenke/Lohoffs (2012) Beobachtungen und Ergebnisse zur räumlichen Orientierung in der Netzkommunikation an. Sie geht methodologisch im Wesentlichen gesprächsanalytisch und interaktionallinguistisch vor (vgl. u. a. Selting/Couper-Kuhlen 2000,2001), ergänzt diesen Ansatz aber auf der theoretischen Ebene um eine phänomenologische Perspektive im Sinne Schütz', Luckmanns und Bergers. Damit soll dem Resümee Dürscheid/Brommers (2007: 17) Rechnung getragen werden.

(ZfAL-Bücker)

\section{Diskussion}

Im vorliegenden Beitrag wurde der Frage nachgegangen, welche funktionalen Züge in deutschsprachigen Einleitungen vorhanden sind und welche Formen intertextueller Bezüge in diesen Zügen auftreten. Die Analyse von fünf nach pragmatischen Kriterien ausgewählten linguistischen Fachzeitschriftenartikeln zeigt, dass Swales CARS-Modell, wie er es 1990 entworfen hat, auch für eine Analyse deutschsprachiger Artikel sinnvoll und nutzbar ist. Die Ergebnisse deuten allerdings darauf hin, dass die bereits von Busch-Lauer (2001) vorgenommen Modifizierungen der sub-moves des Zuges „Nische besetzen“ oder „Vorstellung der eigenen Arbeit“, wie sie diesen Zug benennt, sinnvoll für den deutschsprachigen Raum erscheint. Insbesondere die von Busch-Lauer (2001) vorgeschlagene Teilung in „Vorstellung der Arbeit“ (Ziel, theoretische Grundlagen und Methodik, Hypothesen, Hinweise auf die Textgliederung), ,Darstellung von Material und Methoden der Untersuchung“ und „Definitionen von Termini und Beschreibung von Material und Methode der Untersuchung" erscheint auch durch die vorliegende Analyse bestätigt und sinnvoll.

Nicht immer werden allerdings alle Züge in den untersuchten Einleitungen realisiert: Der funktionale Abschnitt, der offenbar am ehesten entfallen kann, bzw. dem im untersuchten Material sehr unterschiedlich Raum gegeben wird, ist der Zug „Nische etablieren“. Dieser Zug kann vollständig entfallen, in untergeordneten Gliedsätzen realisiert oder über Absätze hinweg hergeleitet werden. Die Bandbreite der Realisierungsformen lässt darauf schließen, dass dieser Zug in deutschsprachigen Einleitungen nicht sehr stark konventionalisiert ist. Insgesamt kann eine starke Schwankung in der Fokussierung festgestellt werden: Im Untersuchungsmaterial gab es drei territoriumslastige Einleitungen, eine nischenlastige und eine vorhabenslastige Einleitung. Will man aus diesen deskriptiven Ergebnissen eine Hypothese für eine quantitative Analyse ableiten, so ließe sich die Behauptung aufstellen, dass deutschsprachige Fachaufsatzeinleitungen eher ,vorhabenslastig“ orientiert sind, d. h. 
dazu tendieren der Vorstellung der eigenen Arbeit viel Raum zu geben (was wiederum mit der oben angesprochenen Ausdifferenzierung in die erwähnten sub-moves zusammenhängt).

Das Vorhandensein aller zentralen Elemente einer Einleitung, wie sie John Swales beschreibt, hängt im untersuchten Material nicht mit der Länge eines Textes zusammen. Im Gegenteil können gerade sehr kurze Einleitung alle Elemente enthalten, während längere Texte ihre Länge oft dadurch erreichen, dass sie einen funktionalen Abschnitt sehr zentral setzen (ZGLLindemann et al., GF-Zima).

Die generellste Aussage, die über die Zitierpraxis in deutschsprachigen Fachaufsatzeinleitungen getätigt werden kann, ist die, dass intertextuelle Verweise in allen Einleitungen vorkommen, dass sie aber keineswegs auf einen Zug und schon gar nicht auf den theoretisch erwarteten Zug „Territorium etablieren“ beschränkt oder fokussiert sind. Im Gegenteil deutet das Datenmaterial darauf hin, dass die intertextuelle Verweistätigkeit eher im Zug „Nische besetzen“ also beim Vorstellen der eigenen Arbeit als relevant erachtet wird. Die Häufigkeit von „Anspielungen“ auf Fachterminologie und theoretische Modelle macht deutlich, dass hier die positive Vernetzung mit konkreten bekannten Theorien und Methoden im Vordergrund steht. Diese Konkretisierung entspricht demnach der Aufgabe des Zuges, das eigene Vorhaben möglichst konkret vorzustellen und in einem Theorie- und Methodennetz zu positionieren. Deutschsprachige Einleitungen fokussieren - um in der Metapher der Textur als Gewebe zu bleiben - das Einweben des eigenen Vorhabens in einen konkreten theoretischen Rahmen und nicht einen breiten Forschungsüberblick über ein Forschungsfeld.

Dass intertextuelle Verweise nicht konzentriert im Zug „Territorium etablieren“ zu finden sind, kann damit erklärt werden, dass die Funktion des Forschungsüberblicks manchmal in ein eigenes, der Einleitung nachfolgendes Kapitel (explizit etwa bei GF-Zima), ausgelagert ist. Dass die nachfolgende Artikelstruktur einen Einfluss auf die rhetorische Struktur der Einleitung hat, zeigt Ling Lin (2014) in ihrer jüngsten Untersuchung englischsprachiger Einleitungen, die nachfolgend zur Einleitung ein eigenes Literaturüberblickskapitel aufweisen. Der von Ling Lin gefundene two-move orientation type, also die Beschränkung auf die Identifizierung des Forschungsinteresses und den Überblick über das eigene Forschungsvorhaben, wenn ein selbständiges Literaturüberblickskapitel folgt, trifft allerdings in dieser Form nur auf ZGL-Lindemann zu. Aber auch in den anderen Artikeln scheint die Einleitung vom Forschungsüberblick „entlastet“, die intertextuelle Verweistätigkeit häuft sich im Zug „Nische etablieren“, wo die Vernetzung der eigenen Vorgangsweise mit bestehende Traditionen und Herangehensweisen dominiert . Ein systematischer Vergleich deutschsprachiger Einleitungen mit und ohne nachfolgendes eigenständiges Literaturüberblickskapitel ist ein wertvolles weiteres Untersuchungsgebiet.

Über den Zusammenhang von Formen des Verweisens und funktionalen Zügen lassen sich anhand der qualitativen Untersuchung nicht mehr als Hypothesen aufstellen, die in einer quantitativen Studie zu überprüfen wären. So scheint das indirekte Zitat eine bevorzugte Form im Zug „Territorium etablieren“ zu sein. Das liegt möglicherweise daran, dass die paraphrasierende Zusammenfassung von unterschiedlichsten Belegstellen in einem Satz am direktesten die Funktion des Forschungsüberblicks übernimmt und dass an dieser Stelle gerne kollektivierende Akteur_innen eingesetzt werden, was eine hohe Informationsdichte ermöglicht und damit platzsparend ist. 
Das direkte Zitat in Form einer Anspielung ist dagegen die zentrale Form des intertextuellen Verweises im Zug „Nische besetzen“. Dieser Verweistyp ermöglicht es ein dichtes Netz aus Begrifflichkeiten zu weben, in das die eigene Forschungsleistung eingearbeitet wird. Während diese Form des direkten Zitats also ein wichtiger Verweistyp in deutschsprachigen Fachaufsatzeinleitungen zu sein scheint, wird das semantisch-integrierte und das freistehende direkte Zitat eher vermieden. Diese Befunde erklären auch die Tatsache, dass die Form der nicht-integrierten Quellverweise wesentlich häufiger benutzt wird als die integrierten: Der Forschungsüberblick wird oftmals in Form einer zusammenfassenden, nicht-berichtenden Paraphrase geliefert und als Akteur_innen werden Kollektivierungen eingesetzt, die nach Nennungen von beispielhaften Belegen in einer semantisch-syntaktisch nicht-integrierten Form verlangen. Das Beispiel der unterschiedlichen Präferenzen für intertextuelle Verweistypen im funktionalen Abschnitt „Nische etablieren“ macht aber auch deutlich, dass die Wahl der Verweistypen von der Relevanz eines Zuges abhängig sein kann: Im Fall des Zuges „Nische besetzen“ legt das Datenmaterial nahe, dass eine elaborierte Ausarbeitung der Lücke unerwartete Formen wie direkte Zitate und Blockzitate nötig macht, weil aus diesen Textstellen sehr detaillierte Perspektiven herausgearbeitet werden.

Die beobachteten und diskutierten Phänomene können abschließend in folgenden acht Hypothesen auf den Punkt gebracht werden:

1. Es gibt territoriumslastige, nischenlastige und vorhabenslastige Einleitungstypen.

2. Es gibt keinen Zug, in dem intertextuelle Verweistätigkeit gehäuft auftritt.

3. Die Dominanz eines Zuges beeinflusst die Häufigkeit intertextueller Verweise.

4. In Fachaufsatzeinleitungen wird bevorzugt die Form der nicht-integrierten Quellverweise genutzt.

5. Das indirekte Zitat ist bevorzugte Form des Zuges „Territorium etablieren“. Diese Zitatform wird gehäuft dafür benutzt, um einen Forschungsüberblick zu liefern.

6. Mit Ausnahme der „Anspielung“ wird das direkte Zitat in der Einleitung eher vermieden.

7. Die „Anspielung“ ist eine zentrale intertextuelle Verweisform im Zug „Nische besetzen“. Diese Verweisform wird dazu genutzt, die eigene Arbeit in ein Forschungsnetz einzubinden.

8. Die Form der intertextuellen Verweise orientiert sich im funktionalen Abschnitt „Nische etablieren“" an der Ausführlichkeit, mit der eine Forschungslücke thematisiert wird.

Abschließend sollen noch einige Einschränkungen der Untersuchung thematisiert werden: Das nach strukturellen und pragmatischen Kriterien zusammengestellte Datenmaterial hat sich hinsichtlich eines Punktes als zufällig homogen erwiesen: Alle Autor_innen haben nach 2010 promoviert, drei sind zum Zeitpunkt der Publikation (Anders et al.) noch gar nicht promoviert. Vor einer Überprüfung der Annahmen in einer umfassenden quantitativen Umsetzung wäre noch an weiteren fünf Artikeln von Wissenschafter_innen mit umfangreicherer Publikationserfahrung zu prüfen, inwieweit die aufgestellten Hypothesen in einem heterogeneren Kontext gelten oder zu modifizieren wären.

Die exemplarische Untersuchung von nur fünf Fachaufsatzeinleitungen lässt bereits vermuten, dass die Kombination von Zitations- und Genreanalyse einen interessanten Ansatzpunkt für die Analyse und Didaktik wissenschaftlichen Schreibens bietet. Aus den Analysen authentischer wissenschaftlicher Artikel können funktionale Verfahrensweisen im Umgang 
mit Intertextualität herausgelesen werden, die als Vorbilder und Lernziele für Noviz_innen eingesetzt werden können.

\section{Literaturverzeichnis}

American Psychological Association (2009): Publication manual of the American Psychological Association (6. ed.). Washington, DC: American Psychological Association. Anders, Christina Ada/Palliwoda, Nicole/Schröder, Saskia (2014): „'in dem moment wo ich es dann erkenne dann ist es auch gleich wieder weg' - Salienzeffekte in der Sprachperzeption“. Linguistik Online 66/4: 51-69.

Atai, Mahmood Reza/Pejman, Habibie (2012): “Genre analysis of applied linguistics research article introductions: Exploring sub-disciplinary variations". Taiwan International ESP Journal 4/1: 25-44.

Bruce, Ian (2014): "Expressing criticality in the literature review in research article introductions in applied linguistics and psychology". English for Specific Purposes 36: 8596.

Bücker, Jörg (2013): „Sinngebiete lebensweltlicher Erfahrung in der SMS- und UsenetKommunikation: Ein Vergleich“. Zeitschrift für angewandte Linguistik 59/1: 21-19.

Busch-Lauer, Ines-Andrea (2001): Fachtexte im Kontrast. Eine linguistische Analyse zu den Kommunikationsbereichen Medizin und Linguistik. Frankfurt a. M./Wien: Lang.

Charles, Maggie (2006): "Phraseological patterns in reporting clauses used in citation: A corpus-based study of theses in two disciplines". English for Specific Purposes 25/3: 310331.

Davis, Mary (2013): "The development of source use by international postgraduate students". Journal of English for Academic Purposes 12/2: 125-135.

Flowerdew, John/Li, Yongyan (2007): „Language Re-use among Chinese Apprentice Scientists Writing for Publication“. Applied Linguistics 28/3: 440-465.

Gnutzmann, Claus/Lange, Regina (1990): „Kontrastive Textlinguistik und Fachsprachenanalyse“. In: Gnutzmann, Claus (ed.): Kontrastive Linguistik. Frankfurt a. M., Lang: 85-112.

Griffig, Thomas (2006) Intertextualität in linguistischen Fachaufsätzen des Englischen und Deutschen. Frankfurt a. M./Wien: Lang.

Gruber, Helmut/Huemer, Birgit/Rheindorf, Markus (2009): Wissenschaftliches Schreiben. Ein Praxisbuch für Studierende der Geistes- und Sozialwissenschaften. Wien: Böhlau.

Gruber, Helmut/Rheindorf, Markus/Wetschanow, Karin/Reisigl Martin/Muntigl, Peter/ Czinglar, Christine (2006): Genre, Habitus und wissenschaftliches Schreiben. Wien: Lit.

Harwood, Nigel (2009): "An interview-based study of the functions of citations in academic writing across two disciplines”. Journal of Pragmatics 41/3: 497-518.

Hirano, Eliana (2009): "Research article introductions in English for specific purposes: A comparison between Brazilian Portuguese and English". English for Specific Purposes 28/4: 240-250.

$\mathrm{Hu}$, Guangwei/Wang, Guihua (2014): "Disciplinary and ethnolinguistic influences on citation in research articles". Journal of English for Academic Purposes 14: 14-28.

Huemer, Birgit/Rheindorf, Markus/Gruber, Helmut (2012): Abstract, Exposé und Förderantrag. Wien: Böhlau. 
Hyland, Ken (1999): "Academic attribution: citation and the construction of disciplinary knowledge". Applied Linguistics 20/3: 341-367.

Hyland, Ken (2000): Disciplinary discourses: Social interaction in academic writing. London: Pearson.

Jakobs, Eva-Maria (1999): Textvernetzung in den Wissenschaften. Zitat und Verweis als Ergebnis rezeptiven, reproduktiven und produktiven Handelns. Tübingen: Niemeyer.

Jakobs, Eva-Maria (2003): "Reproductive writing - Writing from sources". Journal of Pragmatics 35/6: 893-906.

Jalilifar, Alireza/Dabbi, Razieh (2012): "Citation in Applied Linguistics: Analysis of Introduction Sections of Iranian Master's Theses”. Linguistik Online 57/7: 91-104.

Lin, Ling/Evans, Stephen (2012): "Structural Patterns in empirical research articles: A crossdisciplinary study". English for Specific Purposes 31/3: 150-160.

Lindemann, Katrin/Ruoss, Emanuel/Weinzinger, Caroline (2014): „Dialogizität und sequenzielle Verdichtung in der Forenkommunikation: Editieren als kommunikatives Verfahren“. Zeitschrift für germanistische Linguistik 42/2: 223-252.

Ling, Lin (2014): "Innovations in structuring article introductions: The case of Applied Linguistics". Iběrica 28: 129-153.

Mansourizadeh, Kobra/Ahmad, Ummul K. (2011): "Citation practices among non-native expert and novice scientific writers". Journal of English for Academic Purposes 10/3: 152161.

Martín, Pedro/Léon Pérez, Isabel K. (2014): “Convincing peers of the value of one's research: A genre analysis of rhetorical promotion in academic texts". English for Specific Purposes 34: 1-13.

Okamura, Akiko (2008): "Citation Forms in Scientific Texts: Similarities and Differences in L1 and L2 Professional Writing". Nordic Journal of English Studies 7/3: 61-81.

Ozturk, Ismet (2007): "The textual organisation of research article introductions in applied linguistics: Variability within a single discipline”. English for Specific Purposes 26/1: 2538.

Petkova, Mikaela (2012): „Der Kreislauf der Kontaktphänomene“. Sociolinguistica 26/1: 117.

Petkova-Kessanlis, Mikaela (2009): Musterhaftigkeit und Varianz in linguistischen Zeitschriftenaufsätzen. Sprachhandlungs-, Formulierungs-, Stilmuster und ihre Realisierung in zwei Teiltexten. Frankfurt a. M.: Lang.

Petrić, Bojana (2007): "Rhetorical functions of citations in high- and low-rated master's theses". Journal of English for Academic Purposes 6/3: 238-253.

Prestin, Maike (2011): Wissenstransfer in studentischen Seminararbeiten. Rekonstruktion der Ansatzpunkte für Wissensentfaltung anhand empirischer Analysen von Einleitungen. München: Iudicium.

Samraj, Betty (2005): "An exploration of a genre set: Research article abstracts and introductions in two disciplines". English for Specific Purposes 24/2: 141-156.

Samraj, Betty (2008): “A discourse analysis of master's theses across disciplines with a focus on introductions". Journal of English for Academic Purposes 7/1: 55-67.

Samraj, Betty (2013): "Form and function of citations in discussion sections of master's theses and research articles". Journal of English for Academic Purposes 12/4: 299-310. 
Shi, Ling (2010): "Textual Appropriation and Citing Behaviors of University Undergraduates". Applied Linguistics 31/1: 1-24.

Swales, John (1981): Aspects of article Introductions. Birmingham, UK: University of Aston, Language Studies Unit.

Swales, John (1986): “Citation analysis and discourse analysis". Applied Linguistics 7/1: 3956.

Szurawitzki, Michael (2011): Der thematische Einstieg. Eine diachrone und kontrastive Studie auf der Basis deutscher und finnischer linguistischer Zeitschriftenartikel. Frankfurt a. M.: Lang.

Szurawitzki, Michael (2014): „Einleitungen wissenschaftlicher Zeitschriftenartikel aus der DaF-Perspektive: Welche Vorgehensweise ist angemessen? Vorüberlegungen zur Erforschung“. In: Graefen, Gabriele/Moll, Melanie (eds.): Internationale Deutschlehrertagung (IDT) Bozen 2013. Beiträge der Sektion G 6 (Wissenschaftssprache).

Szurawitzki, Michael/Eiglsperger, Thomas (2014): „Makro- und mikrostrukturelle Analysen von Einleitungen wissenschaftlicher Artikel aus dem Deutschen Ärzteblatt“". Glottotheory 5/2: 219-241.

Tessuto, Girolamo (2015): "Generic structure and rhetorical moves in English-language empirical law research articles: Sites of interdisciplinary and interdiscursive cross-over". English for Specific Purposes 37: 13-26.

Thielmann, Winfried (2009): Deutsche und englische Wissenschaftssprache im Vergleich: hinführen - verknüpfen - benennen. Heidelberg: Synchron Wissenschaftsverlag der Autoren.

Thompson, Paul (2005): "Points of focus and position: Intertextual reference in PhD theses". Journal of English for Academic Purposes 4/4: 307-323.

Thompson, Paul/Tribble, Chris (2001): "Looking at citations: Using corpora in English for academic purposes". Language Learning and Technology 5/3: 91-105.

White, Howard D. (2004): "Citation Analysis and Discourse Analysis Revisited". Applied Linguistics 25/1: 89-116.

Zima, E. (2014). „Gibt es multimodale Konstruktionen? Gibt es multimodale Konstruktionen? Eine Studie $\mathrm{zu}$ [V(motion) in circles] und [all the way from X PREP Y]". Gesprächsforschung - Online: Zeitschrift zur verbalen Interaktion 15: 1-48. 